\title{
Formation of Metallic Nanowires by Laser Ablation in Liquid Helium
}

\author{
Victor Lebedev • Peter Moroshkin • \\ Bernard Grobety • Eugene Gordon • Antoine Weis
}

Received: 6 May 2011 / Accepted: 11 July 2011 / Published online: 29 July 2011

(C) Springer Science+Business Media, LLC 2011

\begin{abstract}
Laser ablation of metals in liquid helium results in the formation of metal filaments with diameters on the order of 2-10 nanometres and of spherical nanoparticles. In superfluid helium these nanowires aggregate into centimeter-sized networks. We study the morphology and the electric conductivity of these large aggregates, as well as extinction spectra and the crystalline structure of the individual nanofragments. We discuss the effect of superfluidity on the mechanisms of coalescence processes at the nanometer and centimeter scales.
\end{abstract}

Keywords Superfluid helium · Laser ablation · Nanoparticles · Nanowires · Turbulence

\section{Introduction}

Laser ablation of solid targets immersed in liquids, such as water or ethanol is widely used for producing metallic ( $\mathrm{Au}, \mathrm{Ag}, \mathrm{Cu}$, etc.) nanoparticles and their aggregates $[1,2]$. The particles' shape and size distribution strongly depends on the properties of the solvent, the presence of a surfactant, and the power, wavelength and pulse

This work was supported by grant No. 200020-129831 of the Schweizerischer Nationalfonds and by the LiMat collaboration.

V. Lebedev $(\bowtie) \cdot$ P. Moroshkin · A. Weis

Department of Physics, University of Fribourg, Fribourg, 1700, Switzerland

e-mail: victor.lebedev@unifr.ch

B. Grobety

Department of Geosciences, University of Fribourg, Fribourg, 1700, Switzerland

E. Gordon

Institute of Problems of Chemical Physics RAS, Chernogolovka, 142432, Russia 
duration of the laser radiation. Liquid ${ }^{4} \mathrm{He}$ is a peculiar solvent because of its low temperature, exceptionally low electric polarizability, large heat conductivity, high purity and chemical inertness. In particular the heat conductivity of liquid helium above $T=2.17 \mathrm{~K}(\mathrm{He} \mathrm{I}$ ) is $\approx 0.02 \mathrm{~W} / \mathrm{m} \cdot \mathrm{K}$ (compare to $0.58 \mathrm{~W} / \mathrm{m} \cdot \mathrm{K}$ for water or $0.17 \mathrm{~W} / \mathrm{m} \cdot \mathrm{K}$ for ethanol at room temperature), whereas in the superfluid helium (He II) below $T=2.17 \mathrm{~K}$ the heat flow becomes nonlinear function of the temperature gradient and is several orders of magnitude higher than in He I [3]. Viscosity of $\mathrm{He} \mathrm{I}$ is $\approx 2 \times 10^{-6} \mathrm{~Pa} \cdot \mathrm{s}$ (that is three orders of magnitude smaller than that of water at room temperature) and vanishes in He II. Moreover, superfluid helium has the unique feature of forming quantized vortices which influence the diffusion and coalescence of ablated atoms and clusters.

Although laser ablation (LA) of metals in liquid $\mathrm{He}$ is used in matrix-isolation spectroscopy since the 1990's [4-8], the ablation process is not well studied. In particular the formation of nanoparticles (NP) during the laser ablation of $\mathrm{Cs}$ and $\mathrm{Rb}$ in liquid helium was reported [4, 9]. In such experiments macroscopic filament-like structures have been observed [4, 10-12]. On the other hand the motion of micrometersized solid particles in liquid helium is now being extensively studied by theorists [13-15] and experimentalists $[17,18]$ in the context of quantum turbulence. It was found experimentally [16-18] that solid hydrogen particles in superfluid helium aggregate into extended filaments.

In a recent numerical study of the coalescence of molecules solvated in cryogenic helium fluid [19] the formation of clusters with strongly branched and fractal structures was predicted.

Recently we have reported [11] the first experimental microscopic study of metallic filaments produced by laser ablation in liquid helium. In normal fluid helium, micron-sized particles and nano-networks do not form large complexes and fall onto the bottom of the vessel. In the case of superfluid helium submicron-size particles and nanowires coalesce into macroscopic "ropes". In that publication we have proposed that quantum turbulence can support the macroscopic assembly processes. In the present article we provide a more detailed survey of the ablation process in superfluid and normal fluid helium and of the properties of the aggregation products.

\section{Experimental Set-up}

The experiment was carried out in a helium bath cryostat with optical access. Windows allow us to introduce the ablation laser beam and to perform spectroscopic measurements in situ, and to do visual observations, as well as photographic and video recordings (Fig. 1). The temperature of the liquid helium sample in the cryostat can be reduced by pumping on its free surface. Below $2.17 \mathrm{~K}{ }^{4} \mathrm{He}$ in the bath becomes superfluid (Fig. 2). The lowest achievable temperatures is $1.3 \mathrm{~K}$. The sample is isolated in a thick-walled copper cell (volume of $200 \mathrm{~cm}^{3}$ ) submerged in the liquid helium bath which controls the cell's, and hence the enclosed helium's temperature. The cell is connected to a high pressure helium gas bottle kept at a room temperature, and the pressure can be varied in the range of 1 to 40 bar. Ablation targets are millimetersized pellets of pure gold, copper, or other metals $(\mathrm{Cs}, \mathrm{Ba}, \mathrm{Rb}$ have been studied up 


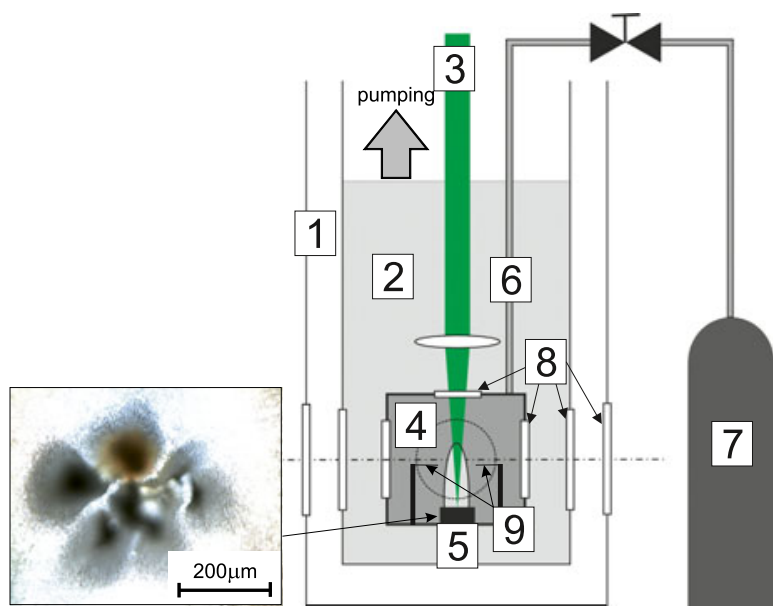

Fig. 1 (Color online) Experimental set-up for laser ablation in condensed helium: 1 isolation vacuum, 2 cooling bath filled with liquid ${ }^{4} \mathrm{He}, 3$ ablation $\mathrm{Nd}$ :YAG laser beam, 4 pressurized helium sample inside the cell, 5 metallic ablation target, 6 thin capillary for cell pressure control, 7 reservoir of pressurized warm helium gas, 8 optical windows, 9 electrodes for conductance measurements. The inset shows a microscopic photograph (picture taken from above along the direction of the ablating laser 3 ) of the craters eroded from the copper target by the ablation laser, each of the 6 craters corresponds to a different laser beam impact spot due to slight beam realignments

Fig. 2 Phase diagram of ${ }^{4} \mathrm{He}$ (after [21])

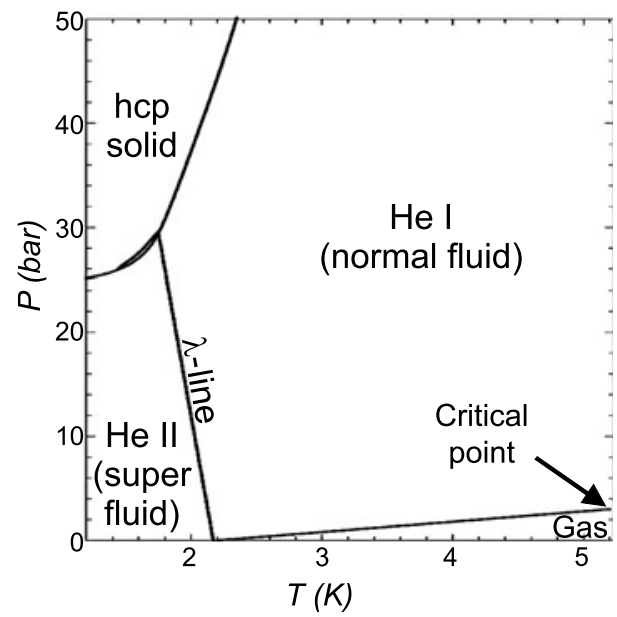

to date) placed on the bottom of the cell. The temperature is measured independently in the bath and in the cell, $\approx 5 \mathrm{~mm}$ away from the target. The pressure is measured in the room temperature part of the filling capillary.

Ablation from the target is performed through the top window. We use the beam of the frequency-doubled $(\lambda=532 \mathrm{~nm})$ or -tripled $(\lambda=355 \mathrm{~nm}) \mathrm{Nd}$ :YAG laser. We can efficiently ablate using $5 \mathrm{~ns}$ long pulses of 40 to $80 \mathrm{~mJ}$ at a repetition rate of 1 to $10 \mathrm{~Hz}$. The laser beam is focused by a $10 \mathrm{~cm}$ focal length lens and hits a spot 
Fig. 3 (Color online) (a) Top view of the cryostat and shadowgraphy set-up: $1 \mathrm{cw}$ diode laser $(632 \mathrm{~nm}), 2$ expanded parallel laser beam, 3 helium bath, 4 sample cell, 5 cryostat, 6 ablation target, 7 projection screen, 8 digital video-camera. (b), (c)

Shadowgraphs of the liquid helium sample (He I) with the ablation laser off (b) and on (c). The focused ablation laser beam is shown by dashed lines in (c)
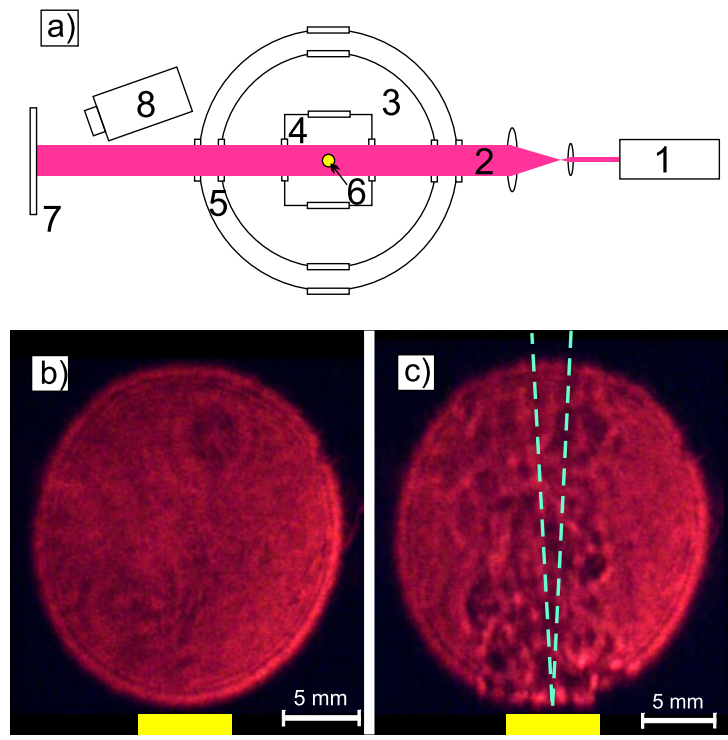

of $\approx 50$ micrometer diameter on the target. The ablation efficiency is very low in comparison with ablation in water by powerful picosecond lasers [20]. From the size of the craters (inset in Fig. 1) created by the laser in the target, we estimate an ablation rate of a few $\mu \mathrm{g} / \mathrm{h}\left(\approx 10^{-10} \mathrm{~g} /\right.$ pulse $)$ during ablation using $10 \mathrm{~Hz}, 80 \mathrm{~mJ}$ pulses of frequency-tripled $\mathrm{Nd}$ :YAG radiation.

Because of the heat deposition on the target by the laser radiation the local temperature above the target may become larger than the bath temperature. Due to the high thermal conductivity of superfluid helium, this heat is efficiently dissipated, and no temperature gradient is created. The medium remains homogeneous and transparent. In normal fluid helium, where the thermal transport is slower, the ablation produces a strongly inhomogeneous distribution of temperature in the sample and a strong convection. Due to the difference of refraction indices of warm and cold liquid helium we can visualize the convective flow by means of shadowgraphy (Fig. 3a). We illuminate the sample by the expanded parallel beam of a cw diode laser. The refraction index inhomogeneity can then be observed as a shadow on a screen behind the cryostat. We use a digital video-camera to continuously monitor the convection flow during the ablation. Selected frames of this recording are shown in Fig. 3b, c.

A pair of pin-shaped electrodes (9 in Fig. 1) was installed in the pressure cell in order to monitor the electrical conductance of the doped helium sample during the ablation. The electrode tips have a radius of $\approx 50 \mu \mathrm{m}$ and a rough surface. A constant voltage of $9 \mathrm{~V}$ was applied to the electrodes and the current through a $1 \mathrm{M} \Omega$ resistor connected in series was monitored. In this low electric field, the ablated ions and field emission from the electrodes produced no measurable signal.

For collecting the ablation products we placed optical microscope slides, graphite substrates and carbon coated copper grids at the bottom of the pressure cell next to the ablation target. These substrates were used for optical microscopy, for secondary 
electron microscopy (SEM), and for transmission electron microscopy (TEM), respectively.

Our standard spectroscopy set-up allows us to record transmission and laser induced fluorescence spectra of the sample in the visible and near infrared range. Transmission spectra were recorded using a halogen lamp and a grating spectrograph equipped with a CCD camera. Laser induced fluorescence of atoms and molecules was excited by the ablation laser and analyzed by the same spectrograph.

In parallel, we monitored the sample through one of the side windows with a high resolution photo-camera and/or video-camera.

\section{Results}

Coalescence of the ablation products into macroscopic filaments was observed for different metals: $\mathrm{Rb}, \mathrm{Cs}, \mathrm{Cu}, \mathrm{Au}, \mathrm{Ba}$. Because of their fast oxidation in air, the filaments and NPs of alkali and alkali-earth metals could not be removed from the cryostat for microscopy investigation. Such studies were therefore only carried out for gold and copper.

\subsection{Optical Spectroscopic Measurements}

We have observed laser induced fluorescence of atoms and molecules produced during ablation in liquid helium. The atomic densities can be estimated from the fluorescence yield to be on the order of $10^{7} \mathrm{~cm}^{-3}$. We have also performed ablation experiments in solid helium at pressures above 25 bar and a temperature of $1.5 \mathrm{~K}$ (Fig. 2). In solid helium the target exposed to the high power laser radiation heats up and melts the helium crystal in a nearby volume of several $\mathrm{mm}^{3}$ (Fig. 1). The ablation products diffuse into the molten volume. When the ablation is stopped, the helium resolidifies thereby immobilizing the dopants. In this way we achieve atomic densities of $\approx 10^{10} \mathrm{~cm}^{-3}$.

Extinction spectrum measurements were done in solid helium, where the clusters and atoms cannot travel to the walls of the cell. Typical light extinction spectra of Csand $\mathrm{Rb}$-doped solid helium samples are shown in Fig. 4. We modeled these spectra assuming that they are due to light scattering by small metallic particles, using fits, based on Lorentz-Mie theory [22]. The fits yield [9] a cluster size distribution centered at about $50 \mathrm{~nm}$ for Cs. The cluster density extracted from the fit is $\approx 10^{10} \mathrm{~cm}^{-3}$. For $\mathrm{Rb}$ we obtain a mean size of $10 \mathrm{~nm}$ and a similar total density. In the case of $\mathrm{Ba}$ we could not observe any formation of clusters, and the extinction spectrum is dominated by the atomic $6 \mathrm{~s}^{2}{ }^{1} S_{0}-6 \mathrm{~s} 6 \mathrm{p}{ }^{1} P_{1}$ absorption line at $540 \mathrm{~nm}$ (Fig. 4), yielding a typical atomic density of $\approx 10^{15} \mathrm{~cm}^{-3}$. We did not observe any extinction in goldand copper-doped samples, although they produce laser induced atomic fluorescence spectra with intensities similar to those of $\mathrm{Cs}$ or $\mathrm{Rb}$.

We suggest that the $\mathrm{Ba}, \mathrm{Cu}$ and Au clusters are very small and cannot be detected by optical means. It is well known [24] that gold NPs with diameters $\leq 3 \mathrm{~nm}$ do not appreciably scatter or absorb light in the visible-NIR range. 
Fig. 4 Transmission spectra of Cs-, Rb- and Ba-doped solid ${ }^{4}$ He samples

Fig. 5 (Color online) Electric conductance of copper-doped liquid He during laser ablation (ablation starts at $t=0$ and stops at $t=950 \mathrm{~s}$ ). The inset shows the first two steps in the conductance change
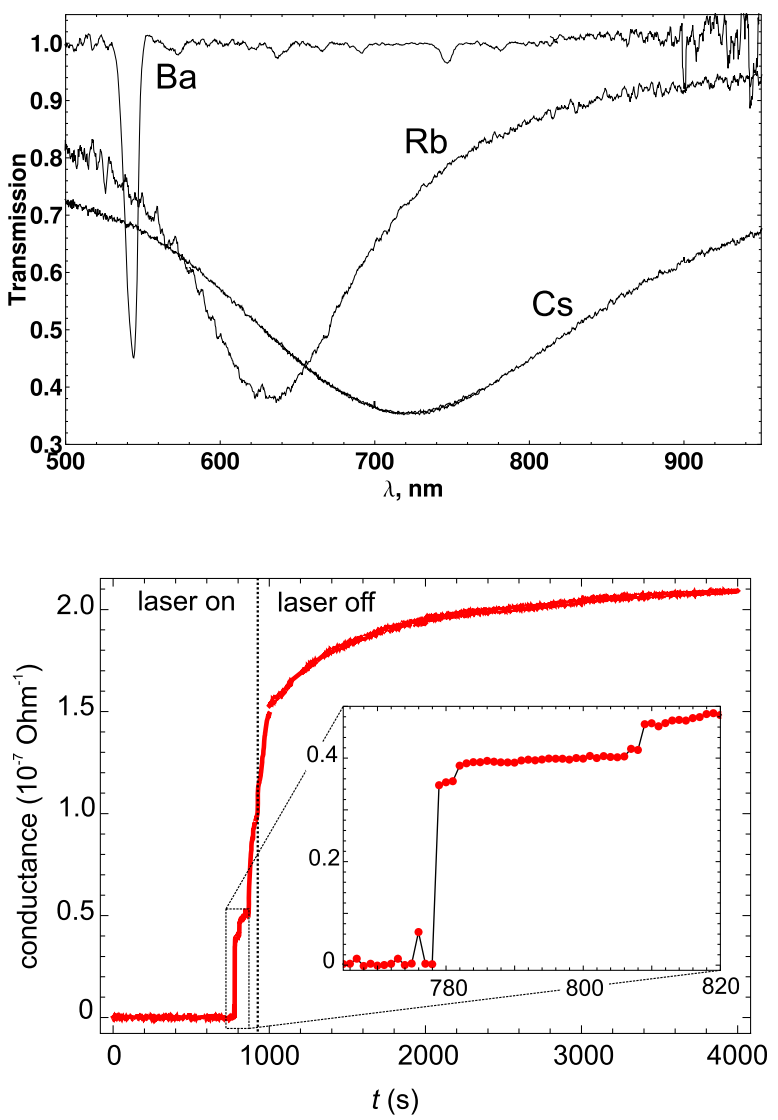

\subsection{Electric Conductivity Measurements and Visual Observations}

As discussed in our first report [11], we have observed stepwise electrical conductivity changes during the ablation in superfluid helium, which set on a few minutes after ablation starts, and which saturates at a level of several $\mathrm{k} \Omega^{-1}$. We related these steps in the conductivity to consecutive electrical connections formed by floating small wires that get stuck to the electrodes and to the cell walls (Fig. 5). Typical step sizes are a few tens of $M \Omega^{-1}$. The conductivity of metal copper at cryogenic temperature is $\approx 2 \times 10^{-11} \Omega \cdot \mathrm{m}$ [23]. A conductance step would thus correspond to filament cross-section on the order of $1 \AA^{2}$, much smaller than the thinnest wires observed with the electron microscope (see Sect. 3.4). Therefore the step sizes were attributed to the finite resistance of the wire/electrode (wire/wall) connections. At the moment of the first conductivity step no filament structures between electrodes can be observed by the naked eye. We conclude that the electrical connections established are due to extended wires of less than a $100 \mu \mathrm{m}$ diameter.

After a few tens of minutes of ablation $(10 \mathrm{~Hz}, 80 \mathrm{~mJ}, 355 \mathrm{~nm})$ one can visually observe extended objects growing from the inner cell surfaces, floating in the sample volume and eventually sticking to the electrodes and the cell body (Fig. 6a). First, 

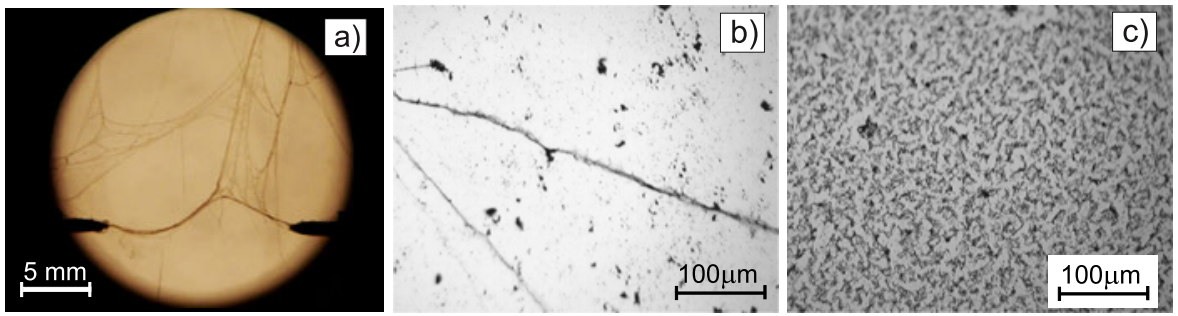

Fig. 6 (Color online) (a) Photograph of the $\mathrm{Cu}$-doped liquid $\mathrm{He}$ sample after several hours of continuous ablation. b, c Microphotographs of the ablated copper collected during the ablation in superfluid He at $1.5 \mathrm{~K}(\mathbf{b})$, in normal fluid $\mathrm{He}$ at $2.6 \mathrm{~K}$ (c)

one sees thin dendrite-like structures developing from the inner surfaces of the cell. As ablation continues these dendrites extend and join together, creating dense "webs" after several hours of ablation. The maximal length of the observed ropes is limited by the inner size of the cell $(\approx 5 \mathrm{~cm})$. The observed ropes are strong enough to survive helium solidification, melting and boiling.

\subsection{Optical Microscopy}

Microscopy studies were only performed with gold and copper. During the ablation and during the warming of the cryostat the nets of the ropes, their fragments and clusters fall onto the substrates at the bottom of the cell. The glass substrates were removed from the cell and the collected material was studied by optical microscopy. Results are shown in Fig. 6b, c. The ablation products from gold and copper targets were found to be very similar. Their morphology strongly depends on the temperature in the cell: in superfluid helium $(T<2.17 \mathrm{~K})$ we obtain mostly extended filaments (ropes) composed of many entangled thinner wires. The longest pieces we collected were $\approx 15 \mathrm{~mm}$ in length and had a rich substructure. The aggregation of the ablation products in normal fluid helium forms highly-structured micron-sized objects that homogeneously cover the substrate surface (Fig. 6c).

\subsection{Electron microscopy}

Secondary electron microscopy (SEM) was used to study the substructure of the metal filaments and microparticles obtained in normal fluid (Fig. 7a-c) and superfluid He (Fig. 7d-f). SEM images show that the macroscopic "ropes" and microparticles are composed of a large number of densely entangled nanometer thick wires with embedded spherical nanoparticles (Fig. 7c, f).

Comparison of the ablation products at high magnification shows that there is virtually no difference in the shapes and sizes of nanowires and nanoparticles, obtained in superfluid (Fig. 7b, c) and in normal fluid (Fig. 7e, f) helium.

It was noticed that the ropes formed in superfluid helium usually have their ends attached to sharp edges, surface defects of the cell walls, etc. For example one can see in Fig. 6a rope attached to the tips of the two electrodes. In Fig. 8 we show SEM images of the surface of the electrode tip, to which the rope was pinned. One can 
Fig. 7 SEM images of the ablation products in superfluid $(\mathbf{a}, \mathbf{b}, \mathbf{c})$ and normal fluid $(\mathbf{d}, \mathbf{e}$, f) $\mathrm{He}$
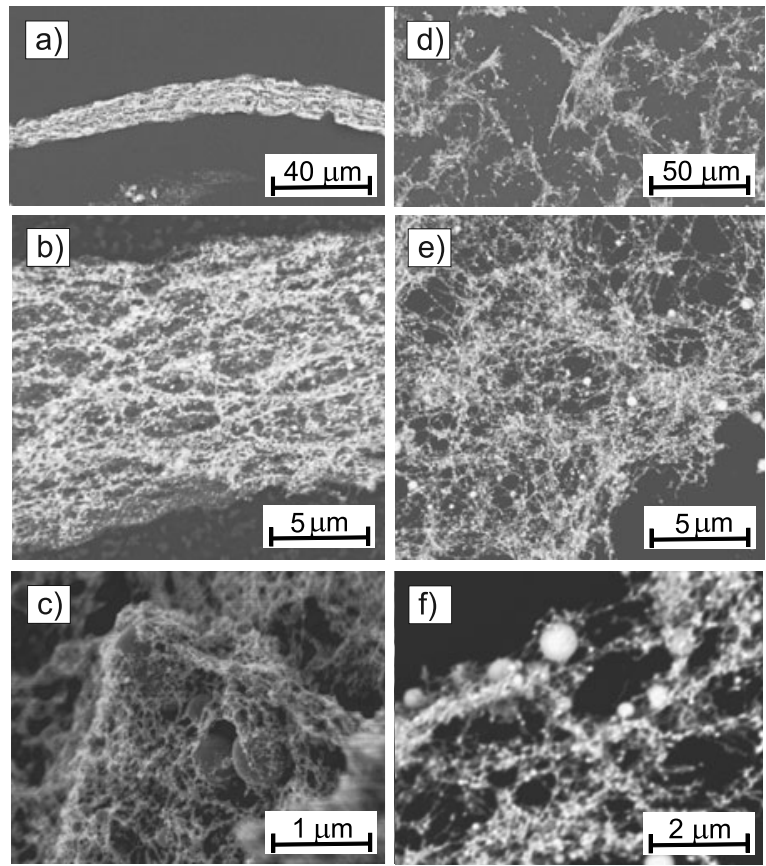

Fig. 8 SEM images of ablation products in superfluid $\mathrm{He}$ on the surface of the electrode
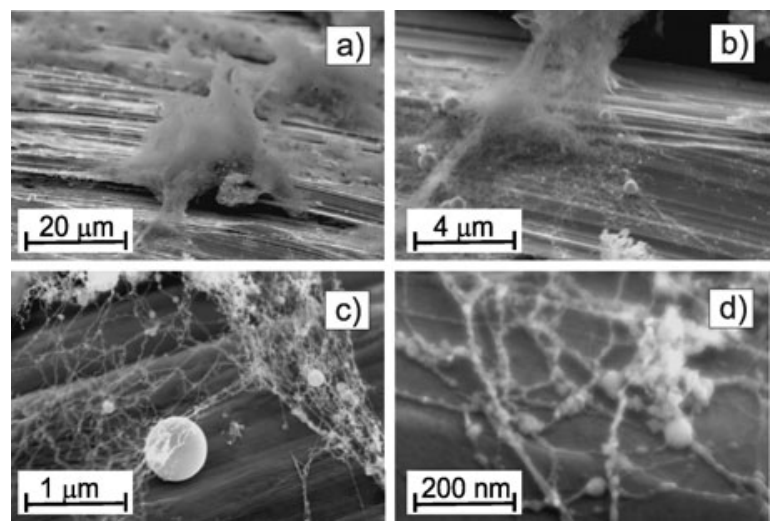

see that the nanowires and nanoparticles preferentially attach to microscopic surface defects.

Transmission electron microscopy images were taken from the solid material that sedimented during and after the experiment onto carbon coated copper grids placed at the bottom of the experimental cell. The gold fragments condensing during the experiment form a net of nanowires together with nanospheres. Nanowires have diameters in the range of 1.5 to $6 \mathrm{~nm}$ (Fig. 9a). The wires are sensitive to the electron beam bombardment in the microscope. The thinnest parts are destroyed which yields typical gaps along the wire. The spherical NPs have diameters ranging from 6 to more than $200 \mathrm{~nm}$. Selected area electron diffraction (SAED) patterns taken with the 


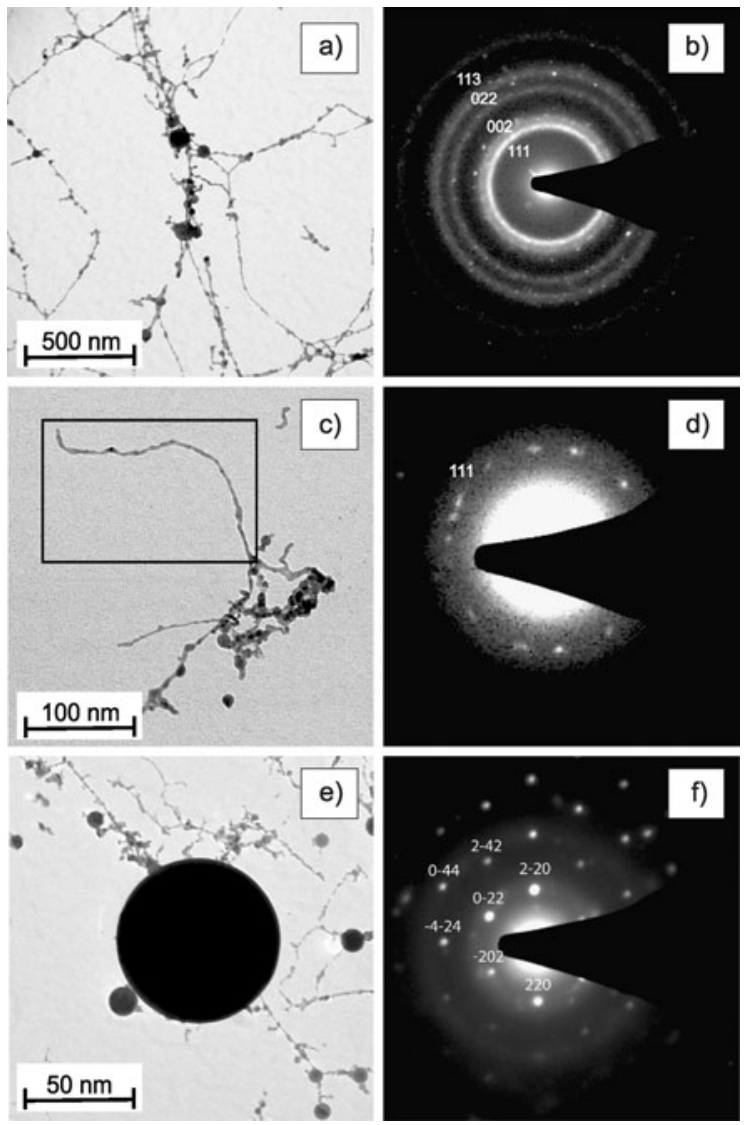

Fig. 9 TEM and corresponding SAED images of the nanowires and nanoparticles collected during and after the ablation in superfluid $\mathrm{He}$

aperture centered over a web-like part of the sample can be indexed for the fcc structure of gold (Fig. 9b). Due to the very small size of the wires, the diffraction rings are broad. SAED patterns with the aperture centered on an individual wire (Fig. 9c) indicate that the wires are polycrystalline (Fig. 9d), whereas the spheres (Fig. 9e) are clearly single crystals (Fig. 9f).

\section{Discussion}

The results of our experiments support the hypothesis suggested earlier $[12,18]$ that nanowires are formed by the coalescence of ablation products that are trapped on the vortices, both in normal fluid and in superfluid helium.

Vortices in superfluid helium have been a topic of research since the 1950's. They have the characteristic feature that the circulation of the He flow around the vortex 
core is quantized [25]

$$
\Gamma=\oint \mathbf{v d s}=\frac{h}{m_{\mathrm{He}}} .
$$

The circulation of helium of density $\rho$ produces a radial pressure gradient at a distance $r$

$$
\frac{\partial p}{\partial r}=\frac{\rho v^{2}(r)}{r}=\frac{\rho \Gamma^{2}}{4 \pi^{2} r^{3}}
$$

that is large enough to trap a metal atom or a nanoparticle near the vortex core $[13,14]$. The trapped particles are strongly confined in the radial direction, but are free to move along the vortex axis. The coalescence of many trapped particles will therefore result in a nanowire with a large aspect ratio. The energy released in the coalescence process is removed by the surrounding superfluid helium. The quantized vortex lines span the full cell volume and are pinned to sharp defects on the walls or may form closed rings. This fact is supported by the observed pinning of the ropes to the cell walls and electrodes.

A similar mechanism must exist also in normal fluid helium, where the turbulent eddies are not quantized, and have a shorter lifetime and a smaller length than the superfluid vortices. The resulting nanowires are hence expected to be shorter and to show no aggregation into macroscopic ropes.

The nanowire formation may thus be catalyzed by the turbulent flow of liquid in the vicinity of the ablation target and in the cell volume, that is due to the temperature gradients induced by the laser deposited heat and by the mechanical vibrations.

In superfluid helium the individual nanowires are twisted together into thick ropes. This phenomenon does not occur in normal fluid helium and is most likely related to the presence of quantized vortices. It is not clear whether a single isolated vortex can be responsible for the build-up of a rope, or whether this process involves a large group of interacting vortices. In fact, numerical studies of superfluid turbulence [26] predict existence of the vortex tangles with structures that strongly resemble the ropes reported here (Fig. 7b).

\section{Conclusion}

The coalescence of the ablation products in liquid helium can be considered as a two-step process. In a first step the nanoparticles and nanofragments are formed on a short timescale close to the ablation spot in the hot environment. In a second step an aggregation of the nanofragments into the wires and ropes in the whole volume of the superfluid helium sample occurs. In normal fluid helium all types of micron-sized fragments are spread uniformly in the sample, since convective and turbulent flows are not regular at a macroscopic scale.

In summary, we have shown that the laser ablation of metals in liquid helium leads to the formation of nanowires, spherical nanoparticles and their aggregation into macroscopic metallic filaments. We suggest that the nanowires are created by turbulent flows in the liquid. The large filament formation is assisted by quantized vortices in superfluid helium. We believe that experiments of this work will give a better insight into the processes of nanoparticle formation. 
Acknowledgements Authors thank J.P. Toennies for stimulating discussions and proposals.

\section{References}

1. A.V. Simakin, V.V. Voronov, N.A. Kirichenko, G.A. Shafeev, Nanoparticles produced by laser ablation of solids in liquid environment. Appl. Phys. A 79, 1127 (2004)

2. F. Mafune, J. Kohno, Y. Takeda, T. Kondow, Formation of gold nanonetworks and small gold nanoparticles by irradiation of intense pulsed laser onto gold nanoparticles. J. Phys. Chem. B 107, 12589 (2003)

3. J. Wilks, The Properties of Liquid and Solid Helium (Clarendon Press, Oxford, 1967)

4. A. Fujisaki, K. Sano, T. Kinoshita, Y. Takahashi, T. Yabuzaki, Implantation of neutral atoms into liquid helium by laser sputtering. Phys. Rev. Lett. 71(7), 1039 (1993)

5. J.H.M. Beijersbergen, Q. Hui, M. Takami, Spectroscopy and dynamics of trapped alkaline earth atoms in superfluid helium. Phys. Lett. A 181, 393 (1993)

6. M. Arndt, R. Dziewior, S. Kanorsky, A. Weis, T.W. Hänsch, Implantation and spectroscopy of metal atoms in solid helium. Z. Phys. B 98, 377 (1995)

7. M. Mudrich, B. Forkl, S. Müller, M. Dvorak, O. Bünermann, F. Stienkemeier, Kilohertz laser ablation for doping helium nanodroplets. Rev. Sci. Instrum. 78, 103106 (2007)

8. P. Moroshkin, A. Hofer, A. Weis, Atomic and molecular defects in solid ${ }^{4}$ He. Phys. Rep. 469, 1 (2008)

9. P. Moroshkin, A. Hofer, S. Ulzega, A. Weis, Impurity-stabilized solid ${ }^{4}$ He below the solidification point of pure helium. Nat. Phys. 3, 786 (2007)

10. M. Arndt, Optische Spektroskopie und Magnetresonanz an Metallatomen in flüssigem und festen (4)Helium. Ph.D. thesis, Ludwig Maximilians Universität München (1995). Max-Planck-Institut für Quantenoptik, Garching, MPQ-Report 197

11. P. Moroshkin, V. Lebedev, B. Grobety, C. Neururer, E.B. Gordon, A. Weis, Nanowire formation by gold nano-fragment coalescence on quantized vortices in he ii. Europhys. Lett. 90, 34002 (2010)

12. E.B. Gordon, A.V. Karabulin, V.I. Matyushenko, V.D. Sizov, I.I. Khodos, Electric properties of metallic nanowires obtained in quantum vortices of superfluid helium. Low Temp. Phys. 36, 590 (2010)

13. N.G. Berloff, P.H. Roberts, Capture of an impurity by a vortex line in a Bose condensate. Phys. Rev. B 63, 024510 (2000)

14. D. Kivotides, C.F. Barenghi, Y.A. Sergeev, Interactions between particles and quantized vortices in superfluid helium. Phys. Rev. B 77, 014527 (2008)

15. M.S. Paoletti, R.B. Fiorito, K.R. Sreenivasan, D.P. Lathrop, Visualization of superfluid helium flow. J. Phys. Soc. Jpn. 77, 111007 (2008)

16. I.F. Silvera, Ultimate fate of a gas of atomic hydrogen in a liquid-helium chamber: Recombination and burial. Phys. Rev. B 29(7), 3899 (1984)

17. G.P. Bewley, D.P. Lathrop, K.R. Sreenivasan, Visualization of quantized vortices. Nature 441, 588 (2006)

18. E.B. Gordon, R. Nishida, R. Nomura, Y. Okuda, Filament formation by impurities embedding into superfluid helium. JETP Lett. 85(11), 581 (2007)

19. S.G. Alves, A.F. Vilesov, S.C. Ferreira, Effects of the mean free path and relaxation in a model for the aggregation of particles in superfluid media. J. Chem. Phys. 130, 244506 (2009)

20. N. Barsch, J. Jacoby, S. Weiler, S. Barcikowski, Pure colloidal metal and ceramic nanoparticles from high-power picosecond laser ablation in water and acetone. Nanotechnology 20(44), 445603 (2009)

21. D.R. Tilley, J. Tilley, Superfluidity and Superconductivity (IOP Publishing, Bristol, 1994)

22. H.C. van de Hulst, Light Scattering by Small Particles (Dover, New York, 1981)

23. D.R. Lide, CRC Handbook of Chemistry and Physics (CRC Press, Cleveland, 1994)

24. M.M. Alvarez, J.T. Khoury, T.G. Schaaff, M.N. Shafigullin, I. Vezmar, R.L. Whetten, Optical absorption spectra of nanocrystal gold molecules. J. Phys. Chem. B 101, 3706 (1997)

25. R.J. Donnelly, Quantized Vortices in Helium II (Cambridge University Press, Cambridge, 1991)

26. S.Z. Alamri, A.J. Youd, C.F. Barenghi, Reconnection of superfluid vortex bundles. Phys. Rev. Lett. 101, 215302 (2008) 